\title{
LIMIT COMPLEXITIES REVISITED
}

\author{
LAURENT BIENVENU ${ }^{1}$, ANDREJ MUCHNIK ${ }^{2}$, ALEXANDER SHEN ${ }^{3}$, \\ AND NIKOLAY VERESHCHAGIN ${ }^{4}$
}

${ }^{1}$ Laboratoire d'Informatique Fondamentale CNRS \& Université de Provence, 39 rue Joliot Curie, F-13453 Marseille cedex 13

E-mail address: Laurent.Bienvenu@lif.univ-mrs.fr

${ }^{2}$ Andrej Muchnik (24.02.1958 - 18.03.2007)

worked in the Institute of New Technologies in Education, Moscow

${ }^{3}$ Laboratoire d'Informatique Fondamentale, Poncelet Laboratory, CNRS, IITP RAS, Moscow

E-mail address: Alexander.Shen@lif.univ-mrs.fr

${ }^{4}$ Moscow State Lomonosov University, Russia

E-mail address: ver@mccme.ru

Abstract. The main goal of this paper is to put some known results in a common perspective and to simplify their proofs.

We start with a simple proof of a result from [7] saying that $\lim \sup _{n} C(x \mid n)$ (here $C(x \mid n)$ is conditional (plain) Kolmogorov complexity of $x$ when $n$ is known) equals $C^{\mathbf{0}^{\prime}}(x)$, the plain Kolmogorov complexity with $\mathbf{0}^{\prime}$-oracle.

Then we use the same argument to prove similar results for prefix complexity (and also improve results of [4] about limit frequencies), a priori probability on binary tree and measure of effectively open sets. As a by-product, we get a criterion of $\mathbf{0}^{\prime}$ Martin-Löf randomness (called also 2-randomness) proved in 3]: a sequence $\omega$ is 2-random if and only if there exists $c$ such that any prefix $x$ of $\omega$ is a prefix of some string $y$ such that $C(y) \geqslant|y|-c$. (In the 1960ies this property was suggested in [1] as one of possible randomness definitions; its equivalence to 2-randomness was shown in [3] while proving another 2-randomness criterion (see also [5]): $\omega$ is 2-random if and only if $C(x) \geqslant|x|-c$ for some $c$ and infinitely many prefixes $x$ of $\omega$.

Finally, we show that the low-basis theorem can be used to get alternative proofs for these results and to improve the result about effectively open sets; this stronger version implies the 2-randomness criterion mentioned in the previous sentence.

Key words and phrases: Kolmogorov complexity, limit complexities, limit frequencies, 2-randomness, low basis.

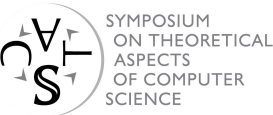

(C) L. Bienvenu, An. Muchnik, A. Shen, and N. Vereshchagin (C) Creative Commons Attribution-NoDerivs License 


\section{Plain complexity}

By $C(x)$ we mean the plain complexity of a binary string $x$ (the length of the shortest description of $x$ when an optimal description method is fixed, see [2]; no requirements about prefixes). By $C(x \mid n)$ we mean conditional complexity of $x$ when $n$ is given [2]. Superscript $\mathbf{0}^{\prime}$ in $C^{\mathbf{0}^{\prime}}$ means that we consider the relativized (with oracle $\mathbf{0}^{\prime}$, the universal enumerable set) version of complexity.

The following result was proved in [7]. We provide a simple proof for it.

\section{Theorem 1.1.}

$$
\limsup _{n \rightarrow \infty} C(x \mid n)=C^{\mathbf{0}^{\prime}}(x)+O(1) .
$$

Proof. We start with the easy part. Let $\mathbf{0}_{n}$ be the (finite) part of the universal enumerable set that appeared after $n$ steps. If $C^{\mathbf{0}^{\prime}}(x) \leqslant k$, then there exists a description (program) of size at most $k$ that generates $x$ using $\mathbf{0}^{\prime}$ as an oracle. Only finite part of the oracle can be used, so $\mathbf{0}^{\prime}$ can be replaced by $\mathbf{0}_{n}$ for all sufficiently large $n$, and oracle $\mathbf{0}_{n}$ can be reconstructed if $n$ is given as a condition. Therefore, $C(x \mid n) \leqslant k+O(1)$ for all sufficiently large $n$, and

$$
\limsup _{n \rightarrow \infty} C(x \mid n) \leqslant C^{\mathbf{0}^{\prime}}(x)+O(1) .
$$

Now fix $k$ and assume that $\lim \sup C(x \mid n)<k$. This means that for all sufficiently large $n$ the string $x$ belongs to the set

$$
U_{n}=\{u \mid C(u \mid n)<k\} .
$$

The family $U_{n}$ is an enumerable family of sets (given $n$ and $k$, we generate $U_{n}$ ); each of these sets has less than $2^{k}$ elements. We need to construct a $\mathbf{0}^{\prime}$-computable process that given $k$ generates at most $2^{k}$ elements, and among them all elements that belong to $U_{n}$ for all sufficiently large $n$. (Then strings of length $k$ may be assigned as $\mathbf{0}^{\prime}$-computable codes of all generated elements.)

To describe this process, consider the following operation: for some $u$ and $N$ add $u$ to all $U_{n}$ such that $n \geqslant N$. (In other terms, we add a horizontal ray starting from $(N, u)$ to the set $\mathcal{U}=\left\{(n, u) \mid u \in U_{n}\right\}$.) This operation is acceptable if all $U_{n}$ still have less than $2^{k}$ elements after it (i.e., if before this operation all $U_{n}$ such that $n \geqslant N$ either contain $u$ or have less than $2^{k}-1$ elements).

For given $u$ and $k$ we can find out using $\mathbf{0}^{\prime}$-oracle whether this operation is acceptable. Now for all pairs $(N, u)$ (in some computable order) we perform $(N, u)$-operation if it is acceptable. (The elements added to some $U_{i}$ remain there and are taken into account when next operations are attempted.) This process is $\mathbf{0}^{\prime}$-computable since after any finite number of operations the family $\mathcal{U}$ is enumerable (without any oracle) and its enumeration algorithm can be $\mathbf{0}^{\prime}$-effectively found (uniformly in $k$ ).

Therefore the set of all elements $u$ that participate in acceptable operations during this process is uniformly $\mathbf{0}^{\prime}$-enumerable. This set contains less than $2^{k}$ elements (otherwise $U_{n}$ would become too big for large $n$ ). Finally, this set contains all $u$ such that $u$ belongs to the (initial) $U_{n}$ for all sufficiently large $n$. Indeed, the operation is always acceptable if all added elements are already present. 
The proof has the following structure. We have an enumerable family of sets $U_{n}$ that have less than $2^{k}$ elements. This implies that the set

$$
U_{\infty}=\liminf _{n \rightarrow \infty} U_{n}
$$

has less than $2^{k}$ elements (the lim inf of a sequence of sets is the set of elements that belong to almost all sets of the sequence). If this set were $\mathbf{0}^{\prime}$-enumerable, we would be done. However, this may be not the case: the criterion

$$
u \in U_{\infty} \Leftrightarrow \exists N(\forall n \geqslant N)\left[u \in U_{n}\right]
$$

has $\exists \forall$ prefix before an enumerable (not necessarily decidable) relation, that is, one quantifier more than we want (to guarantee that $U_{\infty}$ is $\mathbf{0}^{\prime}$-enumerable). However, in our proof we managed to cover $U_{\infty}$ by a set that is $\mathbf{0}^{\prime}$-enumerable and still has less than $2^{k}$ elements.

\section{Prefix complexity and a priori probability}

Now we prove similar result for prefix complexity (or, in other terms, for a priori probability). Let us recall the definition. The function $a(x)$ on binary strings (or integers) with non-negative real values is called a semimeasure if $\sum_{x} a(x) \leqslant 1$. The function $a$ is lower semicomputable if there exists a computable total function $(x, n) \mapsto a(x, n)$ with rational values such that for every $x$ the sequence $a(x, 0), a(x, 1), \ldots$ is a non-decreasing sequence that has limit $a(x)$.

There exists a maximal (up to a constant factor) lower semicomputable semimeasure $m$. The value $m(x)$ is sometimes called the a priori probability of $x$. In the same way we can define conditional a priory probability $m(x \mid n)$ and $\mathbf{0}^{\prime}$-relativized a priori probability $m^{0^{\prime}}(x)$.

\section{Theorem 2.1.}

$$
\liminf _{n \rightarrow \infty} m(x \mid n)=m^{\mathbf{0}^{\prime}}(x)
$$

up to a $\Theta(1)$ factor.

(In other terms, two inequalities with $O(1)$ factors hold.)

Proof. If $m^{\mathbf{0}^{\prime}}(x)$ is greater that some $\varepsilon$, then for some $k$ the increasing sequence $m^{\mathbf{0}^{\prime}}(x, k)$ that has limit $m^{\mathbf{0}^{\prime}}(x)$ becomes greater than $\varepsilon$. The computation of $m^{\mathbf{0}^{\prime}}(x, k)$ uses only finite amount of information about the oracle, thus for all sufficiently large $n$ we have $m^{\mathbf{0}_{n}}(x) \geqslant m^{\mathbf{0}_{n}}(x, k)>\varepsilon$. So, similar to the previous theorem, we have

$$
\liminf _{n \rightarrow \infty} m(x \mid n) \geqslant \liminf _{n \rightarrow \infty} m^{\mathbf{0}_{n}}(x) \geqslant m^{\mathbf{0}^{\prime}}(x)
$$

up to $O(1)$ factors.

In the other direction the proof is also similar to the previous one. Instead of enumerable finite sets $U_{n}$ now we have a sequence of (uniformly) lower semicomputable functions $x \mapsto m_{n}(x)=m(x \mid n)$. Each of $m_{n}$ is a semimeasure. We need to construct a lower $\mathbf{0}^{\prime}$-semicomputable semimeasure $m^{\prime}$ such that

$$
m^{\prime}(x) \geqslant \liminf _{n \rightarrow \infty} m_{n}(x)
$$


Again, the lim inf itself cannot be used as $m^{\prime}$ : though $\sum_{x} \liminf { }_{n} m_{n}(x)<1$ if $\sum_{x} m_{n}(x) \leqslant$ 1 for all $n$, but, unfortunately, the equivalence

$$
r<\liminf _{n \rightarrow \infty} a_{n} \Leftrightarrow\left(\exists r^{\prime}>r\right)(\exists N)(\forall n \geqslant N)\left[r^{\prime}<a_{n}\right]
$$

has too many quantifier alternations (one more than needed; note that lower semicomputable $a_{n}$ makes [...] condition enumerable). The similar trick helps. For a triple $(r, N, u)$ consider an increase operation that increases all values $m_{n}(u)$ such that $n \geqslant N$ up to a given rational number $r$ (not changing them if they were greater than or equal to $r$ ). This operation is acceptable if all $m_{n}$ remain semimeasures after the increase.

The question whether operation is acceptable is $\mathbf{0}^{\prime}$-decidable; if it is, we get a new (uniformly) lower semicomputable (without any oracle) sequence of semimeasures and can repeat an attempt to perform an increase operation for some other triple. Doing that for all triples (in some computable ordering), we can then define $m^{\prime}(u)$ as the upper bound of $r$ for all successful $(r, N, u)$ increase operations (for all $N)$. This gives a $\mathbf{0}^{\prime}$-lower semicomputable function; it is a semimeasure since we verify the semimeasure inequality for every successful increase attempt; finally, $m^{\prime}(u) \geqslant \liminf m_{n}(u)$ since if $m_{n}(u) \geqslant r$ for all $n \geqslant N$, then $(r, N, u)$-increase does not change anything and is guaranteed to be acceptable.

The expression $-\log m(x)$ equals the so-called prefix complexity $K(x)$ (up to $O(1$ ) term; see [2]). The same is true for relativized and conditional versions, an we get the following reformulation of the last theorem:

\section{Theorem 2.2.}

$$
\limsup _{n \rightarrow \infty} K(x \mid n)=K^{\mathbf{0}^{\prime}}(x)+O(1)
$$

Another corollary improves a result of [4]. For any (partial) function $f$ from $\mathbb{N}$ to $\mathbb{N}$ we define the limit frequency of an integer $x$ as

$$
q_{f}(x)=\liminf _{n \rightarrow \infty} \frac{\#\{i<n \mid f(i)=x\}}{n}
$$

In other words, we look at the fraction of $x$-terms in $f(0), \ldots, f(n-1)$ (undefined values are also listed) and take lim inf of these frequencies. It is easy to see that for a total computable $f$ the function $q_{f}$ is a lower $\mathbf{0}^{\prime}$-semicomputable semimeasure. The argument above proves the following result:

Theorem 2.3. For any partial computable $f$ the function $q_{f}$ is upper bounded by a lower $\mathbf{0}^{\prime}$-semicomputable semimeasure.

In 4 it is shown that for some total computable $f$ the function $q_{f}$ is a maximal lower $\mathbf{0}^{\prime}$ semicomputable semimeasure and therefore $\mathbf{0}^{\prime}$-relativized a priori probability can be defined as maximal limit frequency for total computable functions. Now we see that the same is true for partial computable functions: allowing them to be partial does not increase the maximal limit frequency.

The similar argument also is applicable to the so-called a priori complexity defined as negative logarithm of a maximal lower semicomputable semimeasure on the binary tree (see [8]). This complexity is sometimes denoted as $K A(x)$ and we get the following statement:

Theorem 2.4.

$$
\limsup _{n \rightarrow \infty} K A(x \mid n)=K A^{\mathbf{0}^{\prime}}(x)+O(1) .
$$


(To prove this we define an increase operation in such a way that it increases not only $a(x)$ but also $a(y)$ for $y$ that are prefixes of $x$, if necessary. The increase is acceptable if $a(\Lambda)$ still does not exceed 1.)

It would be interesting to find out whether similar results are true for monotone complexity or not (the authors do not know this).

\section{Open sets of small measure}

We now try to apply the same trick in a slightly different situation, for effectively open sets. The Cantor space $\Omega$ is a set of all infinite sequence of zeros and ones. An interval $\Omega_{x}$ (for a binary string $x$ ) is formed by all sequences that have prefix $x$. Open sets are unions of intervals. An effectively open subset of $\Omega$ is an enumerable union of intervals, i.e., the union of intervals $\Omega_{x}$ where $x$ are takes from some enumerable set of strings.

We consider standard (uniform Bernoulli) measure on $\Omega$ : the interval $\Omega_{x}$ has measure $2^{-l}$ where $l$ is the length of $x$.

A classical theorem of measure theory says: if $U_{0}, U_{1}, U_{2}, \ldots$ are open sets of measure at most $\varepsilon$, then $\liminf _{n} U_{n}$ has measure at most $\varepsilon$, and this implies that for every $\varepsilon^{\prime}>\varepsilon$ there exists an open set of measure at most $\varepsilon^{\prime}$ that covers $\liminf { }_{n} U_{n}$.

Indeed,

$$
\liminf _{n \rightarrow \infty} U_{n}=\bigcup_{N} \bigcap_{n \geqslant N} U_{n}
$$

and the measure of the union of an increasing sequence

$$
V_{N}=\bigcap_{n \geqslant N} U_{n}
$$

equals the limit of measures of $V_{N}$, and all these measures do not exceed $\varepsilon$ since $V_{N} \subset U_{N}$. It remains to note that for any measurable set $X$ its measure is the infimum of the measures of open sets that cover $X$.

We now can try to "effectivize" this statement in the same way as we did before. First we started with an (evident) statement: if $U_{n}$ are finite sets of at most $2^{k}$ elements, then $\liminf { }_{n} U_{n}$ has at most $2^{k}$ elements and proved its effective version: for a uniformly enumerable family of open sets $U_{n}$ that have at most $2^{k}$ elements, the set $\liminf _{n} U_{n}$ is contained in a uniformly $\mathbf{0}^{\prime}$-enumerable set that has at most $2^{k}$ elements. Then we did similar thing with semimeasures (again, the non-effective version is trivial: it says that if $\sum_{x} m_{n}(x) \leqslant 1$ for every $n$, then $\left.\sum_{x} \liminf _{n} m_{n}(x) \leqslant 1\right)$.

Now the effective version could look like this. Let $\varepsilon>0$ be a rational number and let $U_{0}, U_{1}, \ldots$ be an enumerable family of effectively open sets of measure at most $\varepsilon$ each. Then for every rational $\varepsilon^{\prime}>\varepsilon$ there exists a $\mathbf{0}^{\prime}$-effectively open set of measure at most $\varepsilon^{\prime}$ that contains $\liminf \operatorname{in}_{n \rightarrow \infty} U_{i}=\bigcup_{N} \bigcap_{n \geqslant N} U_{n}$.

However, the authors do not know whether this is always true. The argument that we have used can nevertheless be applied do prove the following weaker version:

Theorem 3.1. Let $\varepsilon>0$ be a rational number and let $U_{n}$ be an enumerable family of effectively open sets of measure at most $\varepsilon$ each. Then there exists a uniformly $\mathbf{0}^{\prime}$-effectively open set of measure at most $\varepsilon$ that contains

$$
\bigcup_{N} \operatorname{Int}\left(\bigcap_{n \geqslant N} U_{n}\right)
$$


Here $\operatorname{Int}(X)$ denotes the interior part of $X$, i.e., the union of all open subsets of $X$. In this case we do not need $\varepsilon^{\prime}$ (which one could expect since the union of open sets is open).

Proof. Following the same scheme, for every string $x$ and integer $N$ we consider $(x, N)$ operation that adds $\Omega_{x}$ to all $U_{n}$ such that $n \geqslant N$. This operation is acceptable if measures of all $U_{n}$ remain at most $\varepsilon$ for each $n$. This can be checked using $\mathbf{0}^{\prime}$-oracle (if the operation is not acceptable, it becomes known after a finite number of steps).

We attempt to perform this operation (if acceptable) for all pairs in some computable order. The union of all added intervals for all accepted pairs is $\mathbf{0}^{\prime}$-effectively open. If some sequence belongs to the union of the interior parts, then it is covered by some interval $\Omega_{u}$ that is a subset of $U_{n}$ for all sufficiently large $n$. Then some $(u, N)$-operation is acceptable since it actually does not change anything and therefore $\Omega_{u}$ is a part of an $\mathbf{0}^{\prime}$-open set that we have constructed.

\section{Kolmogorov and 2-randomness}

This result has an historically remarkable corollary. When Kolmogorov tried to define randomness in 1960ies, he started with the following approach. A sequence $x$ of length $n$ is "random" if its complexity $C(x)$ (or conditional complexity $C(x \mid n)$; in fact, these requirements are almost equivalent) is close to $n$ : the randomness deficiency $d(x)$ is defined as the difference $|x|-C(x)$ (here $|x|$ stands for the length of $x$ ). This sounds reasonable, but if we then define a random sequence as a sequence whose prefixes have deficiencies bounded by a constant, such a sequence does not exist at all: Martin-Löf showed that every infinite

sequence has prefixes of arbitrarily large deficiency, and suggested a different definition of randomness using effectively null sets. Later more refined versions of randomness deficiency (using monotone or prefix complexity) appeared that make the criterion of randomness in terms of deficiencies possible. But before that, in 1968, Kolmogorov wrote: "The most natural definition of infinite Bernoulli sequence is the following: $x$ is considered $m$-Bernoulli type if $m$ is such that all $x^{i}$ are initial segments of the finite $m$-Bernoulli sequences. MartinLöf gives another, possibly narrower definition" ([1], p. 663).

Here Kolmogorov speaks about " $m$-Bernoulli" finite sequence $x$ (this means that $C(x \mid n, k)$ is greater than $\log \left(\begin{array}{l}n \\ k\end{array}\right)-m$ where $n$ is the length of $x$ and $k$ is the number of ones in $\left.x\right)$. For the case of uniform Bernoulli measure (where $p=q=1 / 2$ ) one would reformulate this definition as follows. Let us define

$$
\bar{d}(x)=\inf \{d(y) \mid x \text { is a prefix of } y\}
$$

and require that $\bar{d}(x)$ is bounded for all prefixes of an infinite sequence $\omega$. It is shown by J. Miller in 3 that this definition is equivalent to Martin-Löf randomness relativized to $\mathbf{0}^{\prime}$ (called also 2-randomness):

Theorem 4.1. A sequence $\omega$ is Martin-Löf $\mathbf{0}^{\prime}$-random if and only if the quantities $\bar{d}(x)$ for all prefixes $x$ of $\omega$ are bounded by a (common) constant.

In turns out that this result (in one direction) easily follows from the previous theorem.

Proof. Assume that $\bar{d}$-deficiencies for prefixes of $\omega$ are not bounded. According to MartinLöf definition, we have to construct for a given $c$ an $\mathbf{0}^{\prime}$-effectively open set that covers $\omega$ and has measure at most $2^{-c}$. 
Fix some $c$. For each $n$ consider the set $D_{n}$ of all sequences $u$ of length $n$ such that $C(u)<n-c$ (i.e., sequences $u$ of length $n$ such that $d(u)>c$ ). It has at most $2^{n-c}$ elements. The requirement $\bar{d}(x)>c$ means that every string extension $y$ of $x$ belongs to $D_{m}$ where $m$ is its length. This implies that $\Omega_{x}$ is contained in every $U_{m}$ where $m \geqslant|x|$ and $U_{m}$ is the set of all sequences that have prefixes in $D_{m}$ (this set has measure at most $2^{-c}$ ). Therefore, in this case the interval $\Omega_{x}$ is a subset of $\bigcap_{m \geqslant|x|} U_{m}$ and (being open) is a subset of its interior. Then we conclude (using the result proved above) that $\Omega_{x}$ (=every sequence with prefix $x$ ) is covered by an $\mathbf{0}^{\prime}$-effectively open set of measure at most $2^{-c}$ constructed as explained above. So if some $\omega$ has prefixes of arbitrarily large $\bar{d}$-deficiency, then $\omega$ is not $\mathbf{0}^{\prime}$ Martin-Löf random.

Note that this argument works also for conditional complexity (with length as condition) and gives a slightly stronger result.

For the sake of completeness we reproduce (from [3]) the proof of the reverse implication (essentially unchanged). Assume that a sequence $\omega$ is covered (for each $c$ ) by a $\mathbf{0}^{\prime}$-computable sequence of intervals $I_{0}, I_{1}, \ldots$ of total measure at most $2^{-c}$. (We omit $c$ in our notation, but all these constructions depend on $c$.)

Using the approximations $\mathbf{0}_{n}$ instead of full $\mathbf{0}^{\prime}$ and performing at most $n$ steps of computation for each $n$ we get another (now computable) family of intervals $I_{n, 0}, I_{n, 1}, \ldots$ such that $I_{n, i}=I_{i}$ for every $i$ and sufficiently large $n$. We may assume without loss of generality that $I_{n, i}$ either has size at least $2^{-n}$ (i.e., is determined by a string of length at most $n$ ) or equals $\perp$ (a special value that denotes the empty set) since only the limit behavior is prescribed. Moreover, we may also assume that $I_{n, i}=\perp$ for $i>n$ and that the total measure of all $I_{n, 0}, I_{n, 1}, \ldots$ does not exceed $2^{-c}$ for every $n$ (by deleting the excessive intervals in this order; the stabilization guarantees that all limit intervals will be eventually let through).

Since $I_{n, i}$ is defined by intervals of size at least $2^{-n}$, we get at most $2^{n-c}$ strings of length $n$ covered by intervals $I_{n, i}$ for given $n$ and all $i$. This set is decidable (recall that only $i$ not exceeding $n$ are used), therefore each string in this set can be defined (assuming $c$ is known) by a string of length $n-c$, binary representation of its ordinal number in this set. (Note that this string also determines $n$ if $c$ is known.)

Returning to the sequence $\omega$, we note that it is covered by some $I_{i}$ and therefore is covered by $I_{n, i}$ for this $i$ and all sufficiently large $n$ (after the value is stabilized), say, for all $n \geqslant N$. Let $u$ be a prefix of $\omega$ of length $N$. All continuations of $u$ of any length $n$ are covered by $I_{n, i}$ and have complexity less than $n-c+O(1)$. In fact, this is a conditional complexity with condition $c$; we get $n-c+2 \log c+O(1)$, so $\bar{d}(u) \geqslant c-2 \log c-O(1)$.

Such a string $u$ can be found for every $c$, therefore $\omega$ has prefixes of arbitrarily large $\bar{d}$-deficiency.

In fact a stronger statement than Theorem 4.1 is proved in [3, 5]; our tools are still too weak to get this statement. However, the low basis theorem helps.

\section{The low basis theorem}

This is a classical result in recursion theory (see, e.g., [6]). It was used in [5] to prove 2-randomness criterion; analyzing this proof, we get theorems about limit complexities as byproducts. For the sake of completeness we reproduce the statement and the proof of low-basis theorem here; they are quite simple. 
Theorem 5.1. Let $U \subset \Omega$ be an effectively open set that does not coincide with $\Omega$. Then there exists a sequence $\omega \notin U$ which is low, i.e., $\omega^{\prime}=\mathbf{0}^{\prime}$

Here $\omega^{\prime}$ is the jump of $\omega$; the equation $\omega^{\prime}=\mathbf{0}^{\prime}$ means that the universal $\omega$-enumerable set is $\mathbf{0}^{\prime}$-decidable.

Theorem [5.1] says that any effectively closed non-empty set contains a low element. For example, if $P, Q \subset \mathbb{N}$ are enumerable inseparable sets, then the set of all separating sequences is an effectively closed set that does not contain computable sequences. We conclude, therefore, that there exists a non-computable low separating sequence.

Proof. Assume that an oracle machine $M$ and an input $x$ are fixed. The computation of $M$ with oracle $\omega$ on $x$ may terminate or not depending on oracle $\omega$. Let us consider the set $T(M, x)$ of all $\omega$ such that $M^{\omega}(x)$ terminates (for fixed machine $M$ and input $x$ ). This set is an effectively open set (if termination happens, it happens due to finitely many oracle values). This set together with $U$ may cover the entire $\Omega$; this means that $M^{\omega}(x)$ terminates for all $\omega \notin U$. If it is not the case, we can add $T(M, x)$ to $U$ and get a bigger effectively open set $U^{\prime}$ that still has non-empty complement such that $M^{\omega}(x)$ does not terminate for all $\omega \in U^{\prime}$. This operation guarantees (in one of two ways) that termination of the computation $M^{\omega}(x)$ does not depend on the choice of $\omega$ (in the remaining non-empty effectively closed set).

This operation can be performed for all pairs $(M, x)$ sequentially. Note that if $U \cup$ $T(M, x)$ covers the entire $\Omega$, this happens on some finite stage (compactness), so $\mathbf{0}^{\prime}$ is enough to find out whether it happens or not, and on the next step we have again some effectively open (without any oracle) set. So $\mathbf{0}^{\prime}$-oracle is enough to say which of the computations $M^{\omega}(x)$ terminate (as we have said, this does not depend of the choice of $\omega$ ). Therefore any such $\omega$ is low (the universal $\omega$-enumerable set is $\mathbf{0}^{\prime}$-decidable). And such an $\omega$ exists since the intersection of the decreasing sequence of non-empty closed sets is non-empty (compactness).

\section{Using the low basis theorem}

Let us show how Theorem 1.1 can be proved using the low basis theorem. As we have seen, we have an enumerable family of sets $U_{n}$ that have at most $2^{k}$ elements and need to construct effectively a $\mathbf{0}^{\prime}$-enumerable set that has at most $2^{k}$ elements and contains $U_{\infty}=\liminf _{n} U_{n}$.

If the sets $U_{n}$ are (uniformly) decidable, then $U_{\infty}$ is $\mathbf{0}^{\prime}$-enumerable and we do not need any other set. The low basis theorem allows us to reduce general case to this special one. Let us consider the family of all "upper bounds" for $U_{n}$ : by an upper bound we mean a sequence $V_{n}$ of finite sets that contain $U_{n}$ and still have at most $2^{k}$ elements each. The sequence $V_{0}, V_{1}, \ldots$ can be encoded as an infinite binary sequence (first we encode $V_{0}$, then $V_{1}$ etc.; note that each $V_{i}$ can be encoded by a finite number of bits though this number depends on $V_{i}$ ).

For a binary sequence the property "to be an encoding of an upper bound for $U_{n}$ " is effectively closed (the restriction $\# V_{n}<2^{k}$ is decidable and the restriction $U_{n} \subset V_{n}$ is co-enumerable). Therefore the low basis theorem can be applied. We get an upper bound $V$ that is low. Then $V_{\infty}=\lim \inf V_{n}$ is (uniformly in $k$ ) $V^{\prime}$-enumerable (as we have said: with $V$-oracle the family $V_{n}$ is uniformly decidable), but since $V$ is low, $V^{\prime}$-oracle can be replaced by $\mathbf{0}^{\prime}$-oracle, and we get the desired result. 
This proof though being simple looks rather mysterious: we get something almost out of nothing! (As far as we know, this idea in a more advanced context appeared in [5].)

The same trick can be used to prove Theorem 2.1; here "upper bounds" are distributions $M_{n}$ with rational values and finite support that are greater than $m(x \mid n)$ but still are semimeasures. (Technical correction: first we have to assume that $m(x \mid n)=0$ if $x$ is large, and then we have to weaken the restriction $\sum M_{n}(x) \leqslant 1$ replacing 1 by, say, 2; this is needed since the values $m(x \mid n)$ may be irrational.)

Theorem 2.4 can be also proved in this way (upper bounds should be semimeasures on tree with rational values and finite support).

As to Theorem 3.1, here the application of the low basis theorem allows us to get a stronger result than before (though not the most strong version we mentioned as an open question):

Theorem 6.1. Let $\varepsilon>0$ be a rational number and let $U_{n}$ be an uniformly enumerable family of effectively open sets, i.e.,

$$
U_{n}=\cup\left\{\Omega_{x} \mid(n, x) \in U\right\}
$$

for some enumerable set $U \subset \mathbb{N} \times\{0,1\}^{*}$. Assume that $U_{n}$ has measure at most $\varepsilon$ for every n. Assume also that $U_{i}$ has "effectively bounded granularity", i.e., all strings $x$ such that $(n, x) \in U$ have length at most $c(n)$ where $c$ is a total computable function. Then for every $\varepsilon^{\prime}>\varepsilon$ there exists a $\mathbf{0}^{\prime}$-effectively open set $W$ of measure at most $\varepsilon^{\prime}$ that contains

$$
\liminf _{n \rightarrow \infty} U_{n}=\bigcup_{N} \bigcap_{n \geqslant N} U_{n}
$$

and this construction is uniform.

Proof. First we use the low basis theorem to reduce the general case to the case where $U$ is decidable and for every $(n, x) \in U$ the length of $x$ is exactly $c(n)$.

Indeed, define an "upper bound" as a sequence $V$ of sets $V_{n}$ where $V_{n}$ is a set of strings of length $c(n)$ such that $U_{n}$ is covered by the intervals generated by elements of $V_{n}$. Again $V$ can be encoded as an infinite sequence of zeros and ones, and the property "to be an upper bound" is effectively closed. Applying the low basis theorem, we choose a low $V$ and add it is an oracle. Since $V^{\prime}$ is equivalent to $\mathbf{0}^{\prime}$, for our purpose we may assume that $V$ is decidable.

Now we have to deal with the decidable case. Let us represent the set $U_{\infty}$ as a union of the disjoint sets

$$
F_{0}=\bigcap_{i} U_{i}, F_{1}=\bigcap_{i \geqslant 1} U_{i} \backslash U_{0}, F_{2}=\bigcap_{i \geqslant 2} U_{i} \backslash U_{1}, \ldots
$$

(for each element $x$ in $U_{\infty}$ we consider the last $U_{i}$ that does not contain $x$ ). Each of $F_{i}$ is (in the decidable case) an effectively closed set (recall than $U_{i}$ is open-closed due to the restriction on $c(i)$ ). Moreover, the $F_{i}$ are pairwise disjoint and the family $F_{i}$ satisfies

$$
\liminf _{n \rightarrow+\infty} U_{n}=\bigcup_{i} F_{i}
$$

and thus

$$
\sum_{i} \mu\left(F_{i}\right)=\mu\left(\liminf _{n \rightarrow+\infty} U_{n}\right)
$$


The measure of each of $F_{i}$ is $\mathbf{0}^{\prime}$-computable, and using $\mathbf{0}^{\prime}$-oracle we can find a finite set of intervals that covers $F_{i}$ and has measure

$$
\mu\left(F_{i}\right)+\left(\varepsilon^{\prime}-\varepsilon\right) / 2^{i+1}
$$

Putting all these intervals together, we get the desired set $W$. So the decidable case (and therefore the general one, thanks to low basis theorem) is completed.

\section{Corollary on 2-randomness}

Theorem 6.1 can be used to prove 2-randomness criterion from [3, 5. In fact, this gives exactly the proof from [5]; the only thing we did is structuring the proof in two parts (formulating Theorem 6.1 explicitly and putting it in the context of other results on limits of complexities).

Theorem $7.1([3,5])$. A sequence $\omega$ is $\mathbf{0}^{\prime}$ Martin-Löf random if and only if

$$
C\left(\omega_{0} \omega_{1} \ldots \omega_{n-1}\right) \geqslant n-c
$$

for some $c$ and for infinitely many $n$.

Proof. Let us first understand the relation between this theorem and Theorem 4.1. If

$$
C\left(\omega_{0} \omega_{1} \ldots \omega_{n-1}\right) \geqslant n-c
$$

for infinitely many $n$ and given $c$, then $\bar{d}(x) \leqslant c$ for every prefix $x$ of $\omega$ (indeed, one can find the required continuation of $x$ among prefixes of $\omega$ ). As we know, this guarantees that $\omega$ is $\mathbf{0}^{\prime}$ Martin-Löf random.

It remains to prove that if for all $c$ we have

$$
C\left(\omega_{0} \omega_{1} \ldots \omega_{n-1}\right)<n-c
$$

for all sufficiently large $n$, then $\omega$ is not $\mathbf{0}^{\prime}$-random. Using the same notation as in the proof of Theorem 4.1, we can say that $\omega$ has a prefix in $D_{n}$ and therefore belongs to $U_{n}$ for all sufficiently large $n$. We can apply then Theorem 6.1 since $U_{n}$ is defined using strings of length $n$ (so $c(n)=n$ ) and cover $U_{\infty}$ (and therefore $\omega$ ) by a $\mathbf{0}^{\prime}$-effectively open set of small measure. Since this can be uniformly done for all $c$, the sequence $\omega$ is not $\mathbf{0}^{\prime}$-random.

Remark. The results above may be considered as special cases of an effective version of a classical theorem in measure theory: Fatou's lemma. This lemma guarantees that if $\int f_{n}(x) d \mu(x) \leqslant \varepsilon$ for $\mu$-measurable functions $f_{0}, f_{1}, f_{2}, \ldots$, then

$$
\int \liminf _{n \rightarrow+\infty} f_{n}(x) d \mu(x) \leqslant \varepsilon
$$

The constructive version assumes that $f_{i}$ are lower semicomputable and satisfy some additional conditions; it says that for every $\varepsilon^{\prime}>\varepsilon$ there exists a lower $\mathbf{0}^{\prime}$-semicomputable function $\varphi$ such that liminf $f_{n}(x) \leqslant \varphi(x)$ for every $x$ and $\int \varphi(x) d \mu(x) \leqslant \varepsilon^{\prime}$. 


\section{References}

[1] Kolmogorov A.N., Logical Basis for Information Theory and Probability Theory. IEEE Transactions on Information Theory, v. IT-14, No. 5, Sept. 1968. (Russian version was published in 1969.)

[2] Li M., Vitányi P., An Introduction to Kolmogorov Complexity and Its Applications, Second Edition, Springer, 1997. (638 pp.)

[3] Miller J., Every 2-random real is Kolmogorov random, Journal of Symbolic Logic, 69(2):555-584 (2004).

[4] Muchnik An.A., Lower limits of frequencies in computable sequences and relativized a priori probability, SIAM Theory Probab. Appl., 1987, vol. 32, p. 513-514.

[5] Nies A., Stephan F., Terwijn S., Randomness, relativization and Turing degrees, Journal of Symbolic Logic, 70(2):515-535 (2005).

[6] Odifreddi P., Classical recursion theory, North-Holland, 1989.

[7] Vereshchagin N. K. Kolmogorov complexity conditional to large integers. Theoretical Computer Science, v. 271 (2002), issues 1-2, p. 59-67.

[8] Zvonkin A.K., Levin L. The complexity of finite objects and the development of the concepts of information and randomness by means of the theory of algorithms. Russian Math. Surveys, 25:6 (1970), p. 83-124. 
